\title{
Does Every Study? Implementing Ordinal Constraint in Meta-Analysis
}

\author{
Julia M. Haaf ${ }^{1} \&$ Jeffrey N. Rouder ${ }^{2}$ \\ ${ }^{1}$ University of Amsterdam \\ ${ }^{2}$ University of California-Irvine
}

Draft version 5, July, 2021. This manuscript was accepted for publication at Psychological Methods.

Julia M. Haaf, Psychological Methods Unit, University of Amsterdam, Amsterdam, Netherlands; Jeffrey N. Rouder, Department of Cognitive Sciences, University of California, Irvine, USA.

We are indebted to Sho Tsuji and Julia Carbajal for letting us use their familiar-word-recognition metaanalysis and for helping us make sense of the interpretation of the findings. We thank Paul Speckman for insightful discussions about variable transformations and meta-analysis. This manuscript and analysis code are available at https://github.com/jstbcs/meta-poor.

Correspondence concerning this article should be addressed to Julia M. Haaf, Postbus 15906, 1001 NK AMSTERDAM, The Netherlands. E-mail: j.m.haaf@uva.nl 


\begin{abstract}
The most prominent goal when conducting a meta-analysis is to estimate the true effect size across a set of studies. This approach is problematic whenever the analyzed studies have qualitatively different results, i.e. some studies show an effect in the predicted direction while others show no effect and still others show an effect in the opposite direction. In case of such qualitative differences, the average effect may be a product of different mechanisms, and therefore uninterpretable. The first question in any meta-analysis should therefore be whether all studies show an effect in the same, expected direction. To tackle this question a model with ordinal constraints is proposed where the ordinal constraint holds each study in the set. This "every study" model is compared to a set of alternative models, such as an unconstrained model that predicts effects in both directions. If the ordinal constraints hold, one underlying mechanism may suffice to explain the results from all studies, and this result could be supported by reduced between-study heterogeneity. A major implication is then that average effects become interpretable. We illustrate the model comparison approach using Carbajal et al.'s (2021) meta-analysis on the familiar-word-recognition effect, show how predictor analyses can be incorporated in the approach, and provide R-code for interested researchers. As common in meta-analysis, only surface statistics (such as effect size and sample size) are provided from each study, and the modeling approach can be adapted to suit these conditions.
\end{abstract}

Keywords: Meta-analysis; Bayesian inference; order-constrained inference 
In meta-analysis it is common to estimate a meta-analytic mean across a corpus of studies, as well as to assess how this mean depends on critical covariates. Consider the question of whether infants can distinguish familiar words from novel words (Carbajal, Peperkamp, \& Tsuji, 2021). Because the data are relatively expensive in infant research, meta-analysis is an important tool to address this question. The meta-analytic mean difference may be estimated between the critical conditions of familiar and novel words, and assessed for the typical dependent variables such as looking times or head turns. Moreover, the variable age serves as a critical covariate for theoretical development and guidance of future studies.

Although the meta-analytic mean difference may seem natural and uncontroversial, we have previously questioned its appropriateness in meta-analytic studies (Rouder et al., 2019). We started with the observation that for many experimental paradigms effects that are positive, zero, or negative correspond to different psychological phenomena. For the familiar-word-recognition effect the three relations - negative, null, positive-correspond to a novelty effect, an invariance, and a habituation effect, respectively. In fact, these three relations may correspond to different stages in early language development (Hallé \& Boysson-Bardies, 1994). During the first few months babies do not distinguish familiar words and rare or novel words, and studies investigating the familiar-word-recognition effect with up to 11 month old infants may expect a zero effect. During the next stage of language development, infants may pay more attention to familiar words than novel ones resulting in an expected positive familiar-word-recognition effect. After around 20 months processing of words changes again, and infants may even pay more attention to novel words (Hallé \& Boysson-Bardies, 1994). This distinction between negative, zero, and positive effects poses a problem for conventional meta-analysis. Consider two studies - both ostensibly run on the same population - that had effect sizes of 0.4 in one study, and of -0.2 in another. How well does the average of 0.1 describe either of the two studies? Can we conclude that there is a small positive familiar-word-recognition effect where babies pay more attention to familiar words than novel words? 
Motivated by the concern that qualitatively different outcomes correspond to different psychological processes, Rouder et al. (2019) recommend assessing the following question: How plausible is it that all true study effects are qualitatively the same, that is, all effects are in the same direction, or that some effects are qualitatively different, that is, they are in opposite directions? The distinction between true and observed effects is critical here. A true effect is a study's underlying latent effect, and if the study had infinitely many observations we could observe this true effect. In reality though, we only collect a limited number of observations, and this limited knowledge introduces sample noise. The observed effect is therefore a combination of the true effect and sample noise. Sample noise increases the variability of a collection of observed effects in that observed effects are more variable than true effects. To separate sample noise from true variability, Rouder et al. (2019) used hierarchical modeling. This approach separates true variability from sample noise, and allows to answer questions about the collection of true effects.

If all true effects are plausibly in the same direction, then the average across these effects is much more interpretable as an overall effect of a common phenomenon. Conversely, if true effects are in opposite directions, the average is uninterpretable, and the data pattern begs for the discovery of critical covariates that explain why some effects are positive and some are negative. This issue is discussed in the clinical literature as quantitative vs. qualitative interactions (Gail \& Simon, 1985; Pan \& Wolfe, 1997). In clinical settings, qualitative interaction refers to the case that one treatment is superior for one patient population, and another treatment is superior for another patient population. As such, the effect between the two treatment conditions is opposite for some patients. In this case, recommendation for treatment has to be qualified by the target population. Gail and Simon (1985) proposed a frequentist likelihood ratio test for qualitative interactions for different treatments across patients. In this test, the null hypothesis is that a treatment is effective for all patient populations. Therefore, if we fail to reject the null hypothesis then this is interpreted as evidence for quantitative rather than qualitative differences. Pan and Wolfe (1997) further developed the test to assess qualitative differences across studies rather than 
patients. Here the focus is on different patient subpopulations instead of different patients within a study. Higgins, Thompson, and Spiegelhalter (2009) use Bayesian estimation methods to assess qualitative interactions. They assess the $95 \%$ posterior prediction interval for a new study's true effect. If the interval includes both positive and negative values then they interpret this outcome as evidence for qualitative interactions. Even though Higgins et al. (2009) developed this test they note significant limitations of it. What is needed is a proper Bayesian test such as Bayes factor model comparison (Higgins et al., 2009, p. 147). In light of Higgins and colleagues' self critique, one of the main contributions of Rouder et al. (2019) is therefore a Bayes factor approach of determining whether a set of studies plausibly share a common phenomenology. They call this the does-every-study meta-analysis.

There are three obvious constrained models to consider: A positive-effects model where all true study effects are constrained to be positive, a negative-effects model where all true study effects are constrained to be negative, and a null model where all study effects are constrained to be exactly zero. The alternative to these constrained models is a mixture where some studies are negative, positive, and null. Rouder et al. (2019) called this mixture the unconstrained model. Knowing whether the unconstrained model holds or whether one of the constrained models hold is critical for understanding any psychological phenomenon, and to determine the required theoretical complexity. The four models will be developed subsequently.

There are a number of difficulties in the approach proposed by Rouder et al. (2019). First, the raw, participant-level data from each study are needed to fit the hierarchical models. While there are some examples of collections of studies where raw data are available (e.g. Ebersole et al., 2016; Corker, Donnellan, Kim, Schwartz, \& Zamboanga, 2017; Wagenmakers et al., 2016), the vast majority of meta-analyses is based on published summary statistics rather than raw data. Second, Rouder et al. (2019) require the same dependent measures across all studies. In many meta-analyses the dependent measures and designs vary across studies. For example, dependent variables may be accuracy in some studies, and response 
times in others. It is unreasonable to assume that all studies in a corpus assess the same items in the same questionnaires. Third, the method by Rouder et al. (2019) is not capable to assess effects of study-level predictor variables. In sum, the model comparison approaches that we previously advocated were developed for many-labs type replications where there is a great deal of uniformity, control, and openness in assessing a single question. It was simply not developed for common meta-analyses where designs and scales vary, and where data extraction relies on published information.

We may consider alternative statistical approaches to Bayes factor model comparison. One alternative is mixture modeling to account for qualitative differences between studies. Schlattmann (2009) discusses this approach for meta-analysis, and the general idea is that several normal distributions are estimated that may differ in mean and variability (p. 169). In our view, however, the approach ignores the relevance of the directionality of effects. All distributions in the mixture model allow for positive and negative effects. Additionally, mixture modeling is notoriously difficult, and many observations (or here, studies) are needed to achieve stable results. Large numbers of studies, however, are relatively rare for metaanalyses in psychology. Another alternative may be a Bayesian estimation approach where we calculate each study's credible intervals and simply count the number of studies where the credible interval is completely negative, overlaps with zero, or is completely positive. We have discussed this approach previously in the context of individual differences (Haaf \& Rouder, 2019; Thiele, Haaf, \& Rouder, 2017). The main drawback of this approach is that there needs to be sufficient resolution for any one study to show a qualitative, directional difference to the other studies. The appeal of the Bayes factor approach is that it treats qualitative directional differences as a global property of the collection of studies - even if we cannot tell which study shows an opposite effect, as long as there is sufficient evidence that some studies might the global model comparison will favor the unconstrained model.

Here, we develop the does-every-study Bayes factor approach for common metaanalysis. We address all three limitations of Rouder et al. (2019) by developing models on 
extant summary statistics. In contrast to Rouder et al. (2019), the current development is widely applicable.

In the next section, we present the collection of formal does-every-study models that we subsequently analyze. Following that, we develop meta-regression approaches to understand whether critical covariates affect the underlying phenomenology. We apply the does-everystudy analysis to meta-analytic data on the familiar-word-recognition effect reported by Carbajal et al. (2021). We discuss the importance of prior settings for Bayesian meta-analysis, limitations of the methods, and future directions of does-every-study modeling.

\section{Does-Every-Study Models}

The first step of analysis is to specify what constitutes the data. This choice is limited by the information available in published articles. We decided to use the Fisher's $Z$ effect-size statistic which is typically used to assess the size of a correlation coefficient. There exist easy transformations from Fisher's $Z$ to other popular effect-size statistics such as Cohen's $d$ or $\log$ odds ratio (and also reverse transformations). Fisher's $Z$ is a variance stabilizing transformation of the bivariate correlation coefficient $r$ that maps from the $(-1,1)$ interval into the real number space:

$$
Z=\frac{1}{2} \log \left(\frac{1+r}{1-r}\right)=\operatorname{arctanh}(r)
$$

For those unfamiliar with the statistic Fisher's $Z$, the relationship to the correlation coefficient is a sigmoidal function that is $Z=-\infty$ for $r=-1, Z=0$ for $r=0$, and $Z=\infty$ for $r=1$. The green solid line in Figure 1A shows this relationship. The relationship is similar in character to that between $r$ and Cohen's $d$ (orange, long-dashed line), and indeed Fisher's $Z$ and Cohen's $d$ share the same space from $-\infty$ to $\infty$. Importantly, the key property for our analysis, the direction of the effect, is preserved across effect size measures. Yet, there are critical differences, and Fisher's $Z$ is always smaller in magnitude, sometimes 
much smaller in magnitude than Cohen's $d$.

The advantage of Fisher's $Z$ is that, in the large-sample limit, the distribution of $Z$ is known and convenient for modeling effects across studies that use either continuous or categorical covariates:

$$
Z \sim \operatorname{Normal}\left(\theta, \frac{1}{N-3}\right)
$$

where $\theta$ is the true effect size and $N$ is the number of observations. Importantly, the variance of $Z$ is only dependent on $N$, and is not affected by the size of the correlation. Equation 2 is typically derived for bivariate correlations of two continuous variables. Yet, we also wish to use the approach for experimental settings where one of the variables is discrete. It is well-known in the statistical literature that Equation 2 also holds for the biserial correlations (Gayen, 1951), and for educational purposes we provide a proof in the online supplement.

To address qualitative sameness and differences, we start out similar to common meta-analysis. Models are placed on the collection of study effects much like in what is often called random-effects meta-analysis. For the following models, let $i$ denote the study, $i=1, \ldots, I$. The models are hierarchical in nature, and the first level for all models is

$$
Z_{i} \mid \theta_{i} \sim \operatorname{Normal}\left(\theta_{i}, \frac{1}{N_{i}-3}\right)
$$

where $\theta_{i}$ is the $i$ th study's true $Z$-value. The second level of each model completes the specification on $\theta_{i}$ with hyperpriors. We follow the common approach perhaps stated most clearly by Vanpaemel (2010) and Vanpaemel and Lee (2012) of placing substantive constraints as different prior specifications. Here, these constraints are ordinal and equality constraints. In the last step we specify the third level of the models by placing prior settings. 
The Unconstrained Model. The unconstrained model is a standard linear model without constraints, and it corresponds to the typical random-effects meta-analytic model. Here, the collection of study effects simply follows a normal distribution:

$$
\mathcal{M}_{u}: \quad \theta_{i} \mid \mu, \tau^{2} \stackrel{i i d}{\sim} \operatorname{Normal}\left(\mu, \tau^{2}\right),
$$

where $\mu$ is the mean effect and $\tau^{2}$ is the variance of effects. No constraints are placed on the collection of $\theta_{i}$ such that effects for some studies may truly be positive while effects for other studies may truly be negative.

The Positive-Effects Model. The positive-effects model corresponds to the hypothesis that every study has a true effect in the same, expected direction:

$$
\mathcal{M}_{p}: \quad \theta_{i} \mid \mu, \tau^{2} \stackrel{i i d}{\sim} \operatorname{Normal}_{+}\left(\mu, \tau^{2}\right)
$$

where $\mathrm{Normal}_{+}$is a normal distribution truncated below at zero. The positive domain of the model translates to the idea that effects are coded so that positive is the expected direction. This model statement implies multiple ordinal constraints, one for each study in the data set, reducing model complexity drastically compared to the unconstrained model. Assuming the same mean and variance as the general model, the positive-effects model has higher density for (small) positive effects and no mass below zero.

The Common-Effect Model. The common-effect model corresponds to the frequentist fixed-effect meta-analytic model (Borenstein, Hedges, Higgins, \& Rothstein, 2010). For the common-effect model, all $\theta_{i}$ have the same value:

$$
\mathcal{M}_{c}: \quad \theta_{i} \mid \mu=\mu, \quad \mu>0
$$

Here, the common effect, $\mu$, is restricted to be in the expected direction, just like in the positive-effects model. 
The common-effect model may seem unlikely, and there has been much philosophical and statistical debate about the usefulness of meta-analytic common-effect models (e.g. Hedges \& Vevea, 1998). Surely, there must be some variability between true effects across studies. Yet, especially in meta-analysis, this model may be a necessary addition to the set of models proposed. Borenstein et al. (2010) note, for example, that between study variability may be hard to estimate with small numbers of studies in a data set. Also, the common effect assumption may be meaningful when the experiments where pre-planned to use the exact same methods across labs. This pre-planning is common in medical research, and it recently gained attention in psychological research with the many-labs projects (Ebersole et al., 2016). In fact, Rouder et al. (2019) report evidence for this model for Ebersole and colleagues' many-labs study on moral credentialism.

The Null Model. The last model proposed is a null model. Here, all $\theta_{i}$ are exactly zero:

$$
\mathcal{M}_{0}: \quad \theta_{i}=0
$$

This model is the most constrained model of the set, and it is a strict null in that it does not allow for true variability around zero. Instead, all variation must be captured in the noise term $\frac{1}{N_{i}-3}$. If an effect truly does not exist, then all studies truly should have a null result. True variation around zero would imply that there has to be a trade-off between the studies such that all true effects sum to zero, which strikes us as highly unlikely.

Prior Settings. Because the above second-level specifications are conditional on the mean and variance parameters $\mu$ and $\tau^{2}$, we now discuss prior settings on these parameters. We chose conjugate prior distributions: 

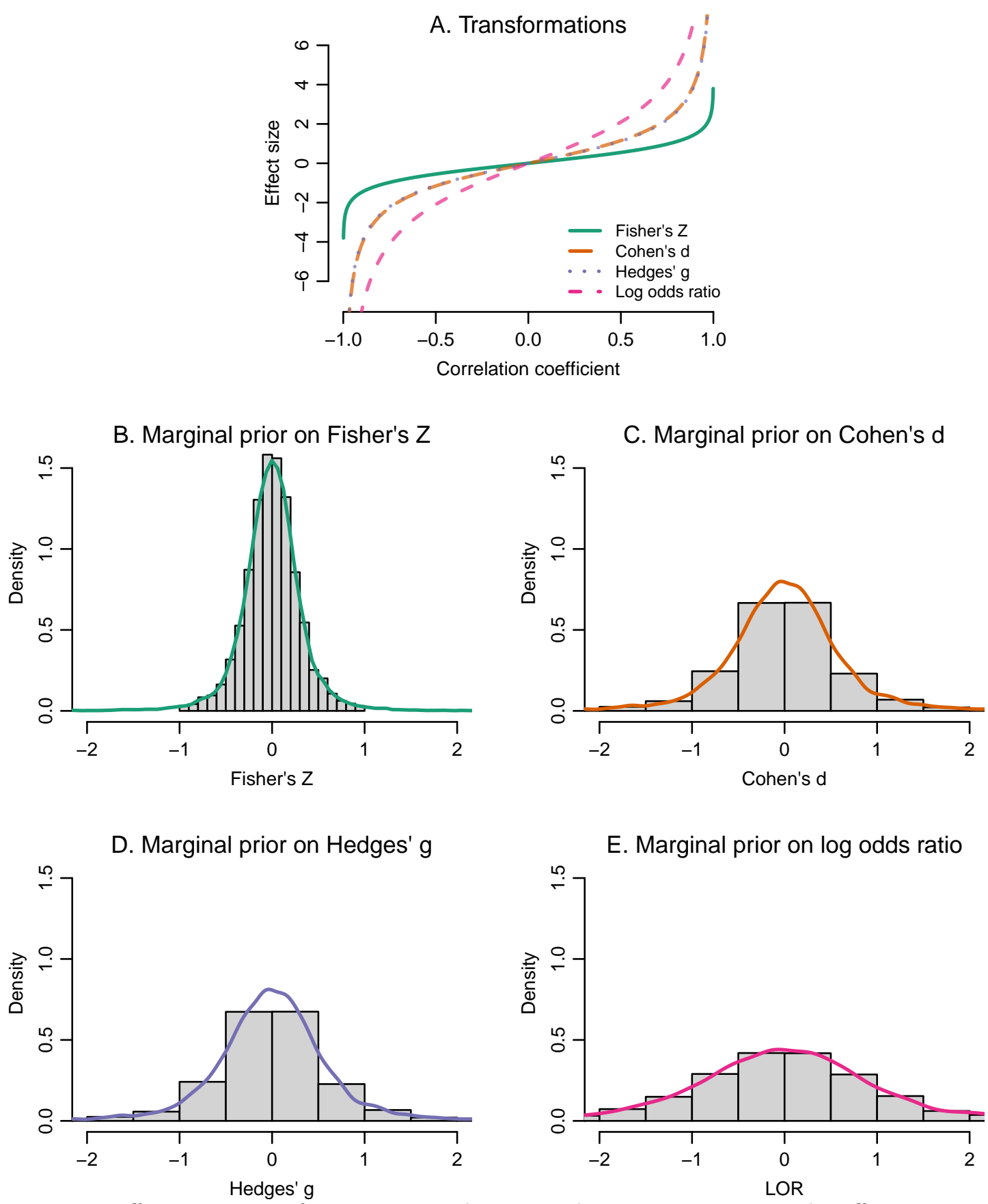

Figure 1. Effect size transformations and marginal priors on true study effect sizes. A. The relationship between the correlation coefficient and different effect size measures. For the same data, effect size measures differ in scale with Fisher's $Z$ corresponding to the smallest scale and Log odds ratio corresponding to the largest scale. B. Marginal prior for any one study's true Fisher's $Z$ statistic. C.-E. Marginal priors for true alternative effect size measures of any one study using conventional transformations. For the same prior settings the scales are drastically different. 

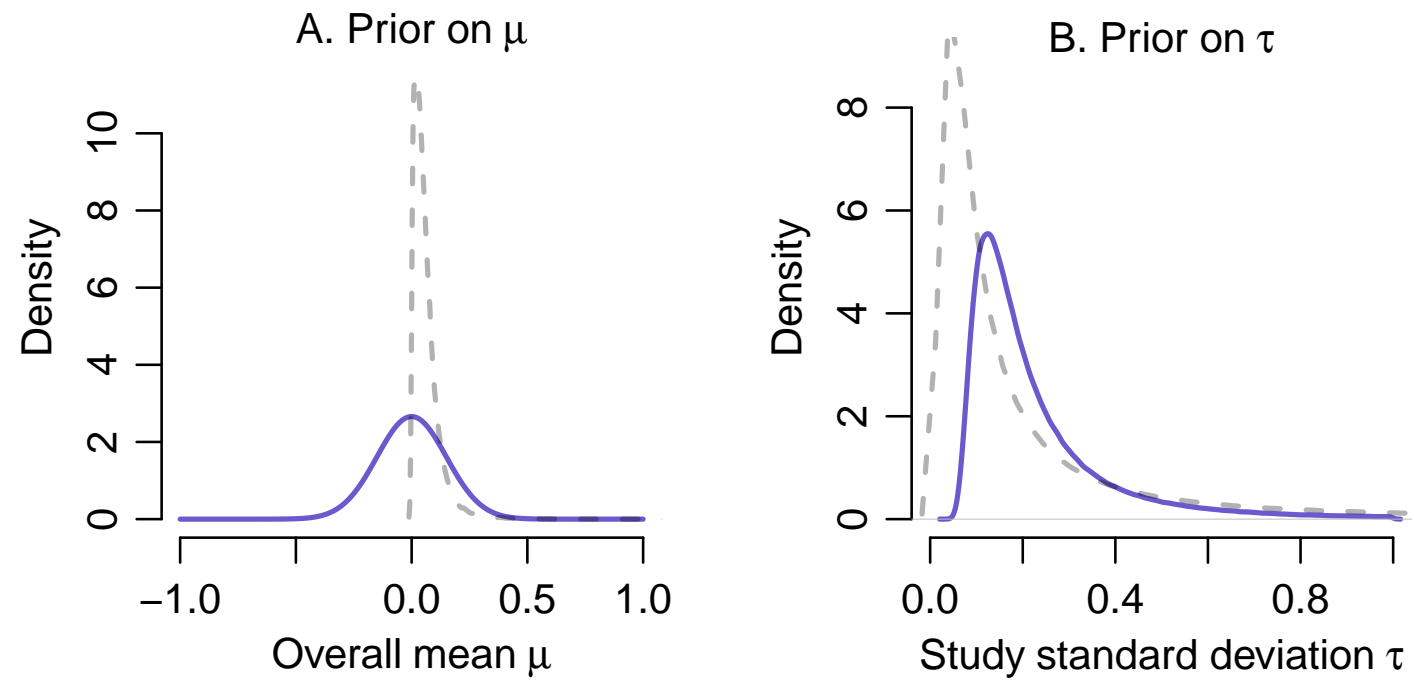

Figure 2. Prior distributions on the overall effect size $\mu(\mathrm{A})$ and between-study standard deviation $\tau$ (B) in units of Fisher's $Z$. The solid lines correspond to the chosen settings, the dashed lines correspond to empirically informed prior settings transformed to Fisher's $Z$ units.

$$
\begin{aligned}
\mu & \sim \operatorname{Normal}\left(0, c_{1}^{2}\right), \\
\tau^{2} & \sim \text { Inverse-Gamma }\left(1, c_{2}^{2}\right) .
\end{aligned}
$$

Here, we set $c_{1}=0.15$ and $c_{2}=0.15$ (see Figure 2 for the prior distributions). These settings can be interpreted as scale settings on the overall effect size $\left(c_{1}\right)$ and the betweenstudy heterogeneity $\left(c_{2}\right)$, and they are in Fisher's $Z$ units. These prior settings are critical for model comparison because they differ between the models. For example, the common-effect model constrains $\tau^{2}$ to zero. Therefore, the prior settings have to be chosen with some care. One way of understanding these priors is to see their consequence on individual-study Fisher-z statistics. The priors on $\mu$ and $\tau^{2}$ may be integrated to form a marginal prior on $\theta_{i}$, and this marginal is shown in Figure 1B. We chose the priors on $\mu$ and $\tau$ so that values of $\theta_{i}$ between -0.5 and 0.5 are most plausible under the unconstrained model. This prior corresponds to a relatively informative distribution while still allowing for the possibility of untypically large effect sizes. 
We believe that the current settings are appropriate for many applications in psychology. There are two additional considerations that may illuminate these choices of prior settings. The first consideration is that the settings are in Fisher's $Z$ units, however, psychologists commonly have more intuition for Cohen's $d$ as effect size measure than Fisher's $Z$. As shown in Figure 1 Fisher's $Z$ scores are always smaller in magnitude than Cohen's $d$. Hence, the priors need to be smaller when compared to possible priors on Cohen's $d$. We discuss the differences between effect size measures and the resulting prior choices in the next section. The second consideration is whether these priors well represent results in the previous literature. Here we briefly discuss one empirical prior for the study standard deviation, $\tau$, and one prior based on prior elicitation for the overall effect size, $\mu$, and assess how these settings compare to our choices. For $\tau$, Erp, Verhagen, Grasman, and Wagenmakers (2017) gathered variability estimates from over 700 meta-analyses on mean differences in the field of psychology, and fit a distribution to the histogram of these estimates. This approach results in the distribution $\tau \sim$ Inverse-Gamma(1,0.15). For ease of comparison, we transformed this distribution to Fisher's $Z$ units and plotted it as the dashed gray line in Figure 2B. As can be seen, the empirical approach results in a distribution with more mass for smaller variability, and perhaps a slightly fatter tail. For $\mu$, the informative prior was obtained using a prior elicitation procedure (Gronau, Ly, \& Wagenmakers, 2019). The resulting prior distribution, $\mu \sim$ Truncated-t $(3,0.35,0.102)$, is again on mean difference. The dashed line in Figure 2A corresponds to the transformed distribution in Fisher's $Z$ units. Again, the prior has much more mass at smaller values.

In the light of this analysis, we are assured that the choices of our prior settings are in the correct range and result in modestly informed priors. We would consider the priors from empirical results and prior elicitation we discuss here as a starting point for good prior choices that perhaps are a bit too narrow. We think more diffuse priors are probably a better approach. Of course, we invite researchers to come to their own conclusions and adjust these prior settings to fit their rationale and their applications. In addition to this $a$ priori assessment of prior settings, we may also assess whether varying prior settings in a 
reasonable range leads to reasonable variability of Bayes factor model comparison results using a sensitivity analysis. We provide more discussion on the issue and a sensitivity analysis subsequently.

Implied Priors on Other Effect Size Measures. In the previous section we discuss prior settings for Fisher's $Z$ effect size estimates as dependent variable. The emphasis on Fisher's $Z$ is not too surprising because prior settings should typically be made with close attention to the scaling of the dependent variable (Haaf \& Rouder, 2017; Rouder, Haaf, \& Aust, 2018). However, the random variable here is effect size, and one might think the scaling is obvious. Yet, as shown in Figure 1A different measures of effect size have different characteristics, and an intuition for expected ranges of, say Cohen's $d$ may not be helpful when developing models for Fisher's $Z$. To better understand these range differences, Figure 1 explores implied priors from our priors on Fisher's $Z$ for several effect size measures commonly used in psychology: Cohen's $d$, Hedges' $g$, and log odds ratio (LOR). Both the curves Figure 1A and the histograms in Figure 1C-E are based on common transformations between the effect size measures (Borenstein, Cooper, Hedges, \& Valentine, 2009).

What do the different characteristics between effect size measures in Figure 1A imply for our choice of prior? To assess this implication, we drew samples from the priors on Fisher's $Z$, and transformed these samples. The resulting histograms represent the marginal prior for any one study's Fisher's $Z$ effect size transformed to the other effect size measures. Panels C-E in Figure 1 show these transformed priors on the alternative measures. The range of implied priors drastically differs across effect size measures. The distributions of Cohen's $d$ and Hedges' $g$ are about twice as wide as the distribution of Fisher's $Z$, and the distribution of log odds ratio is approximately four times as wide as the distribution of Fisher's $Z$. In other words, our priors on Fisher's $Z$ imply quite wide and moderately informed priors on other effect size measures.

There are additional implications in case researchers wanted to model Cohen's $d$ or another effect size measure instead of Fisher's $Z$. The prior settings we recommend 
are in Fisher's $Z$ units and therefore may not be appropriate for other applications. This implication requires a bit of rethinking from frequentist meta-analysis where the same models can be used regardless of the effect size measure (so long as it is continuous and has full support). There are two possibilities on how to achieve the same implied prior settings across different effect size measures. First, researchers may transform their effect size statistic to Fisher's $Z$ and use the prior settings proposed here. Or, second, researchers may adjust prior settings of $c_{1}$ and $c_{2} \cdot{ }^{1}$ For example, if Cohen's $d$ is used instead of Fisher's $Z$, one might try to double the scales $c_{1}$ and $c_{2}$.

\section{Model Comparison}

The main purpose of the modeling approach is to draw inference about the distribution of study effects. To do so, we propose a Bayes factor model comparison approach to compare the unconstrained model, the positive-effects model, the common-effect model, and the null model. Here, we provide a very brief, informal discussion of Bayes factors and the approaches used to estimate them. We have previously provided more extensive discussions in Haaf and Rouder (2017) and Rouder et al. (2019) that were based on Jeffreys (1961), Kass and Raftery (1995), Morey, Romeijn, and Rouder (2016), and Rouder, Morey, and Wagenmakers (2016).

In Bayesian model comparison, the main target of interest is the relative evidence for one model compared to another. The Bayes factor is this relative evidence, and it results directly from Bayes rule. We may also view the Bayes factor as predictive accuracy of one model over another model (Rouder \& Morey, 2019). In this sense, the Bayes factor denotes how well the first model predicted the observed data compared to the other model. Figure 3 illustrates this point. Panel A shows the model specifications for the positive-effects and the unconstrained model for any one study's true effect. The predictions for observed effect sizes

\footnotetext{
${ }^{1}$ Additionally, note that the variance of the distribution of, say Cohen's $d$ is not $\frac{1}{N-3}$. For between-subject designs it is $V_{d}=\frac{n_{1}+n_{2}}{n_{1} \times n_{2}}+\frac{\delta^{2}}{2\left(n_{1}+n_{2}-2\right)}$, where $n_{1}$ and $n_{2}$ are the sample sizes of the two conditions, and $\delta$ is the true effect size. Therefore, the variance of observed effect sizes in Equation (3) needs to be adjusted as well.
} 

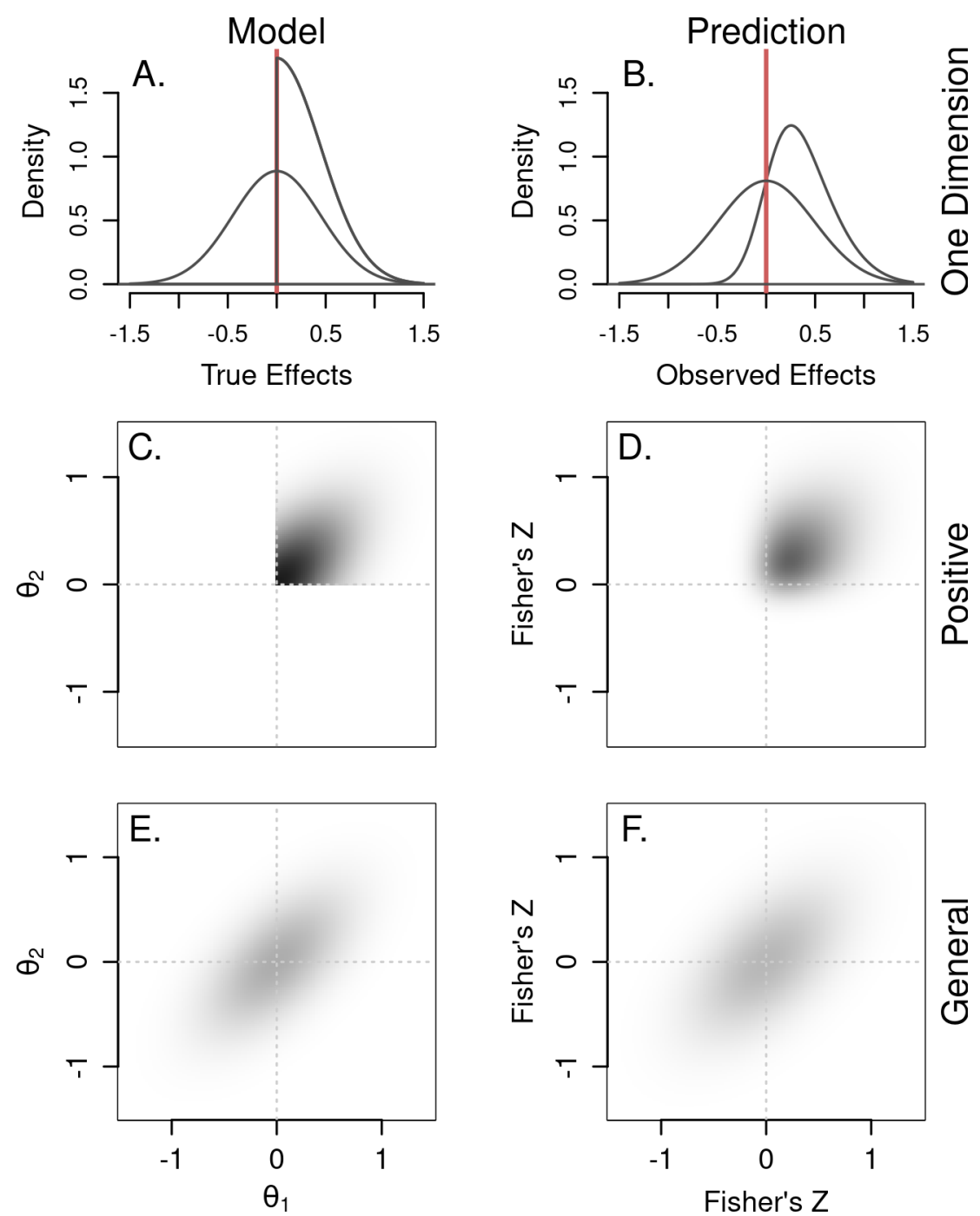

Figure 3. Model specification and predictions for the positive-effects model and the general model. The models are on the left hand-side, and the predictions for data are on the right hand-side. Top row: Models for one study. Even though the positive-effects model is restricted to positive values in specification, it can predict small negative observed values. Middle row: Positive-effects model for two studies. Effects for any two studies are predicted to be correlated due to the hiearchical nature of the model, and mostly positive. Bottom row: General model for two studies. Effects are still correlated, but may be positive or negative. The predictions for any specific effect combination is weaker for the general model as is covers more parameter space. 
from these models are illustrated in panel B. As can be seen, the positive-effects model best predicts positive effects while the general model predicts both positive and negative effects to the same degree. As a result, if a positive effect is observed, the positive-effects model will be preferred as it has more density for positive effects than the general model. In contrast, if a negative effect is observed the general model will be preferred. Yet, the positive-effects model can predict small negative effects despite the ordinal constraint on true effects.

How do the model predictions extend to more than one study effect? Figure 3 shows multivariate model specifications for two studies for both the unconstrained and the positiveeffects model. Panels $\mathrm{C}$ and $\mathrm{D}$ show model specification and model prediction for the positive-effects model. The effect size for Study 1 is specified on the x-axis; the effect size for Study 2 is specified on the y-axis. The correlation between the two effects is introduced by the hierarchical nature of the models (i.e. the common variability of the mean effect). This correlation is also preserved in the predictions, and the positive-effects model best predicts small, similar, positive effects. The predictions of the unconstrained model are shown in Figure 3F. Conceptually, Bayes factors can be understood as a comparison between the prediction in panels $\mathrm{D}$ and $\mathrm{F}$ in Figure 3. If we observed effects for the two studies, say $z_{1}=.2$ and $z_{2}=.25$, then we may compare the predictive accuracy for the point $[.2, .25]$ of these two panels. The ratio of the predictive accuracies at the observed data point is the Bayes factor between the two models.

Practically, Bayes factors can be estimated in several ways. Here, we use the encompassing approach to estimate the Bayes factor between the unconstrained model and the positive-effects model (Haaf \& Rouder, 2017; Hoijtink, 2012; Klugkist, Laudy, \& Hoijtink, 2005), and an analytic approach to assess the Bayes factor between the unconstrained model, the common-effect model, and the null model (Rouder, Morey, Speckman, \& Province, 2012). The Bayes factors between the positive-effects model, the common-effect model, and the null model can be obtained using the transitivity property of Bayes factors. ${ }^{2}$

\footnotetext{
${ }^{2}$ Using transitivity, the Bayes factor between the positive-effects model and the null model, $B_{+0}$, may be obtained as $B_{+0}=\frac{B_{g 0}}{B_{g+}}$.
} 
The encompassing approach employed here is based on Hoijtink (2012), and we have employed and described the approach in Haaf and Rouder (2017), Haaf and Rouder (2019), and Rouder et al. (2019). More interesting is the analytic approach employed here. Because we use surface statistics (such as Fisher's $Z$ ) and their standard errors as data, we derived the analytic Bayes factor between the unconstrained model and the common-effect model, and between the unconstrained model and the null model from scratch. The main target of the approach is the probability of data conditional on each of the two models marginalized over the parameter space. This probability is typically called the marginal probability of a model, and it may be expressed using The Law of Total Probability as

$$
P(\boldsymbol{Y} \mid \mathcal{M})=\int_{\boldsymbol{\xi} \in \Xi} P(\boldsymbol{Y} \mid \boldsymbol{\xi}) P(\boldsymbol{\xi}) d \boldsymbol{\xi}
$$

where $\boldsymbol{\xi}$ is a vector of parameters from parameter space $\Xi$. The likelihood function, $P(\boldsymbol{Y} \mid \boldsymbol{\xi})$, is given by Equation (3), and it is the product of normal densities with mean $\theta_{i}$ and variance $\frac{1}{n_{i}-3}$ evaluated for the data.

Here, we illustrate the analytic approach for obtaining the Bayes factor between the null model and the unconstrained model. For the null model, obtaining the marginal probability is straight-forward. The parameter space of $\theta_{i}$ is reduced to a vector of zeros. The integral in Equation (4) is simply the likelihood of the data when $\theta_{i}=0$ for all $i$. For the unconstrained model, the integral in Equation (4) may be simplified by integrating out the collection of $\theta_{i}$ and the overall effect $\mu$. The likelihood of $Z_{i}$ marginal over $\theta_{i}$ and $\mu$ is

$$
Z_{i} \mid \tau^{2}, \sigma_{\mu}^{2} \sim \operatorname{Normal}\left(0, \frac{1}{n_{i}-3}+\tau^{2}+\sigma_{\mu}^{2}\right)
$$

where $\tau^{2}$ is the variance of $\theta_{i}$ and $\sigma_{\mu}^{2}$, the variance of $\mu$ that is here fixed at $.15^{2}$. The integral is now reduced to the dimension of $\tau^{2}$. To estimate $P(\boldsymbol{Y} \mid \mathcal{M})$ the integral can be evaluated for possible values of $\tau^{2}$ based on its prior distribution. The marginal probability can be estimated in similar fashion for the common-effect model. 


\section{Application: The Familiar-word-recognition Effect}

We are now ready to apply the does-everyone meta-analysis to data. Here, we reanalyzed the meta-analysis on the familiar-word-recognition-effect conducted by Carbajal et al. (2021). The authors gathered 34 studies from 16 research articles studying infants with average sample age ranging from 5 to 15 months. Here, null, positive, and negative effects correspond to different stages in early language development (Hallé \& Boysson-Bardies, 1994). If infants do not distinguish between familiar and unfamiliar words then the familiarword-recognition effect is zero. If infants pay more attention to familiar words than novel ones then the familiar-word-recognition effect is positive. If infants pay more attention to novel words than familiar words then the familiar-word-recognition effect is negative. The expectation is that infants in the age range of the current studies show a positive familiar-word recognition effect. The analysis by Carbajal et al. (2021) showed an overall effect size in line with this expectation, $\hat{\mu}=0.21,95 \% \mathrm{CI}=[0.13,0.3]$. Yet, do all studies plausibly show a qualitatively similar effect, that is, is there one underlying phenomenology?

We first estimated the unconstrained model. The observed and estimated study effect sizes (Fisher's $Z$ ) are shown in Figure 4. The gray squares correspond to the observed effect sizes, and the black points correspond to the estimates from the unconstrained model (posterior means). The gray error bars show the $95 \%$ confidence intervals for Fisher's $Z$. The black error bars show $95 \%$ credible intervals. The size of the points is determined by the study weights that are in turn dependent on the sample size. These weights determine the impact of each study on the overall effect size. As can be seen, there is a substantial amount of hierarchical shrinkage reducing the variability of estimated study effects as compared to observed study effects. The amount of shrinkage for each study is a function of the standard error of the effect size estimate, which in turn is a function of the sample size. Because the studies in the current meta-analysis have relatively small sample sizes-between 12 and 53 participants - as is typical for developmental research, much hierarchical shrinkage is expected towards the overall effect. 


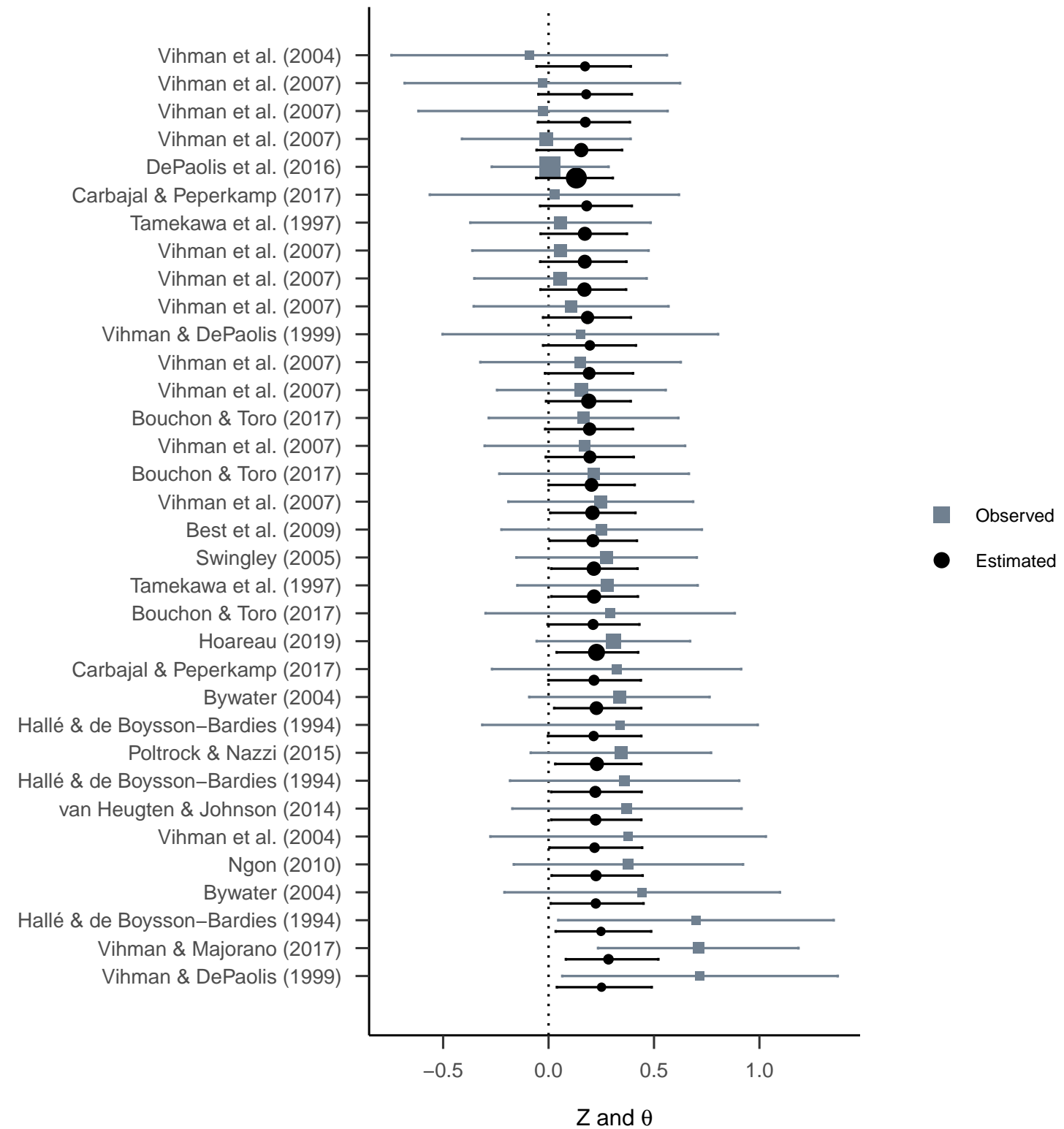

Figure 4. Results for the familiar-word-recognition effect from the unconstrained model. Observed effects with $95 \%$ confidence intervals are depicted by the gray squares and bars, posterior estimates with $95 \%$ credible intervals are given by the black points and bars. There is hierarchical shrinkage to the overall effect reducing the variability of study effects drastically. 
Figure 4 also shows that while three studies have an observed negative effect size the posterior means for all 34 studies are positive. To quantify the evidence for or against every-study-does, we compare the models previously proposed using Bayes factors. The preferred model is the positive-effects model, and it is preferred over the common-effect model by 3 to 1 , over the null model by 9 to 1 , and over the unconstrained model by 16 to 1 (see Table 1). Hence, while there is some evidence that all studies show a positive effect, there is only modest evidence that study effects actually differ in true value.

In summary, at this point, it seems that all studies conform with the expectation that infants pay more attention to novel words. Hence, we may tentatively hold onto the notion that all studies are indeed tapping the same psychological processes and belong functionally to a common phenomenology. We find relatively small differences across estimated true effects. Given the common phenomenology we now feel confident to interpret the overall effect across studies, estimated from the unconstrained model as $\hat{\mu} \mid \boldsymbol{Z}=0.20,95 \% \mathrm{CrI}$ $=[0.12,0.29] .{ }^{3}$ This estimate can be used to inform the design of future studies.

\section{Accounting for Predictors}

A key goal in meta-analysis is to assess the relationship between a predictor variable and the effect size of a dependent measure across studies (Borenstein, Hedges, Higgins, \& Rothstein, 2011). This goal is especially relevant when there is substantial variability of effect sizes. The hope then is that this variability can be explained by one or more predictors. In the usual case, the researcher is concerned about the effect of the predictor on the meta-analytic mean, and fairly straightforward extensions of linear regression may be used to assess whether a predictor accounts for substantial variability. The consideration of predictors, however, is far more nuanced in a does-every-study framework.

In meta-analysis, it is common to consider predictors that act on what we call the study level. These predictors apply to the study as a whole. Examples could be elements of

\footnotetext{
${ }^{3}$ Even though the unconstrained model is not the preferred model we may use it as an estimation model with few theoretical constraints. Note that the estimate would be comparable if we used the positive-effects or the common-effect model.
} 
the experimental design, choice of dependent variable, the age of the target population, or the language of the participants. In keeping with the theme of the development, models are specified for the case where researchers have summary-level data for each study and the values of the predictors for each study.

There are two main targets of inquiry: (a) is the inclusion of a predictor warranted, and (b) if so, does the inclusion change whether there are qualitative or quantitative differences in the set of studies. The main approach here is to specify a set of models that encompass these targets and compare them with Bayes factors.

\section{Meta-regression Models}

The meta-regression model for a single predictor is:

$$
\begin{aligned}
Z_{i} & \sim \operatorname{Normal}\left(\theta_{i}, \frac{1}{N_{i}-3}\right) \\
\theta_{i} & =\nu_{i}+x_{i} \beta
\end{aligned}
$$

where $x_{i}$ denotes the predictor for the $i$ th study, and $\beta$ denotes the slope. The parameter $\theta_{i}$ serves the same role in the previous models without a predictor. To avoid identifiability issues, without any loss of generality we normalize the collection of $x_{i}$ such that the mean is zero and the variance is 1 . Note that in this model, the parameter $\nu_{i}$ combines both a non-zero intercept and residuals. It is not a classic intercept parameter inasmuch as there is one such parameter for each study. It is not a classic residual as it is not zero-centered. The reason for this specification is simple - the model reduces to the previous unconstrained model without predictors when the slope parameter, $\beta$ is zero. Hence, it is a proper generalization of the previous development. With the understanding that the collection of $\nu \mathrm{s}$ is not zero-centered, we will refer to them as the residuals.

Priors are needed on $\beta$ and the collection of $\nu_{i}$ s. For $\beta$ we chose a weakly informative, 
zero-centered prior:

$$
\beta \sim \operatorname{Normal}\left(0,0.15^{2}\right)
$$

For $\nu_{i}$ we kept the prior structure previously shown in the Section "Prior Settings":

$$
\begin{aligned}
\nu_{i} & \sim \operatorname{Normal}\left(\mu, \tau^{2}\right), \\
\mu & \sim \operatorname{Normal}\left(0,0.15^{2}\right), \\
\tau^{2} & \sim \operatorname{Inverse-Gamma}(1,0.02) .
\end{aligned}
$$

\section{Submodels and Interpretations}

The critical question is on the placement of order constraints. In the previous section, we discussed four different models on the true study effects - all were null, all were constant, all were positive, and a lack of constraint. In the regression model, a comparable target is the parameter $\nu_{i}$, the residual effect after accounting for the predictor. To indicate these models, we extend the previous notation as follows. The model $\mathcal{M}_{0+\beta}$ is a null model where the predictor is included and the residuals at the study level are all zero. The models $\mathcal{M}_{c+\beta}$, $\mathcal{M}_{p+\beta}$, and $\mathcal{M}_{u+\beta}$, the common residuals, positive residuals, and unconstrained residuals models, are defined likewise. Note that some of these models are much more plausible than others. For example, a model where all residuals are identically zero seems unreasonable. Therefore, we carry the two models we consider most plausible and informative, $\mathcal{M}_{p+\beta}$ and $\mathcal{M}_{u+\beta}$.

These regression models are defined by constraint on the residuals. Yet, we can also place constraint on the marginal or total effect, $\theta_{i}$. And, surprisingly, the constraint on the marginals and on the residuals may have different implications. The following cases highlight the differences and their theoretical interpretation:

i. Explaining Qualitative Differences. Consider the case where the total effect $\theta_{i}$ violates 
the positive constraints, that is, there is evidence that at least some $\theta_{i}$ are negative. Here, we may conclude that the phenomenon is complex and disunified. Now, suppose that when the predictor is added, the resulting residuals, $\nu_{i}$, obey positive constraints. In this case, the rich results in the total is explained by the predictor. That is, the predictor resulted in converting qualitative differences among the studies to quantitative ones. This case, in our view, represents an advancement of knowledge - the predictor clearly explains why some studies are positive and others are negative.

ii. Robust Quantitative Differences. One facet of inference with Bayes factor is that it is calibrated by the resolution of the data (Rouder, Speckman, Sun, Morey, \& Iverson, 2009). When the data are noisy or few in number, simpler models are supported over more complicated ones. In the context of this report, the null model is simplest, followed in order by the common model, the positive model, and the unconstrained model. An effect of calibration is possible for the familiar-word-recognition data because relatively few studies are included in the meta-analysis. Adding a predictor that accounts for noise effectively increases the resolution of the data. In some cases, if the predictor is added, it may account for variation, and the net result is that the data now have the resolution to support more complex models, say there is evidence for violations of constraint on the residuals that was previously unavailable. Perhaps even more importantly, the same constraint that was obeyed in the total may still hold when predictors are added. If there is still evidence that all studies show a true positive effect, then every-study-does holds even in light of increased resolution. The constraint is robust to the predictor.

We expand the notation as follows. The model where there is constraint on residuals $\nu_{i}$ but not on totals $\theta_{i}$ are denoted as above. The models where there is constraint on $\theta_{i}$ are indicated with parentheses as follows. The model $\mathcal{M}_{(p+\beta)_{p}}$ denotes the constraints where both $\theta_{i}$ and $\nu_{i}$ are positive. To see if the finding is robust to the covariate, we may check that $\mathcal{M}_{(p+\beta)_{p}}$ is superior to all other models. For example, to $\mathcal{M}_{(p+\beta)_{u}}$, where with increased 
resolution from the covariate, we observe violations in the positivity of the marginals. Note that $\mathcal{M}_{(p+\beta)_{u}}$ and $\mathcal{M}_{p+\beta}$ are the same model as they have a constraint on the residuals but not the marginals.

\section{The Familiar-word-recognition Example}

For the familiar-word-recognition meta-analysis, we assess the effect of the average age of study samples on the effect size. To do so, we standardize age and estimate the model using Gibbs sampling. Figure 5A comes from the previous analysis where there is no predictor. One of the things we can see here is shrinkage to the grand mean. Figure 5B now shows estimates for the meta-regression model with the predictor included. The gray open points again reference the observed effect sizes, and the blue points are the estimates for $\theta_{i}$ from the model including the predictor. Notice that the pattern of shrinkage is different: Observed effects are shrunken towards the regression line instead of the grand mean. The slope of the estimated regression line is $\beta=0.06,95 \% \mathrm{CI}=[-0.03,0.14]$. This slope is slightly reduced from the least square error regression line (dashed line). This reduction is due to hierarchical shrinkage of study effect sizes.

Figure $5 \mathrm{~B}$ shows the effect of the predictor on the marginal estimates of the effect. But it does not indicate whether the predictor is evidenced. For that Bayes factors are needed. Table 1 shows Bayes factors for all models as compared to the unconstrained model. As a reminder, $\mathcal{M}_{u+\beta}$ refers to the model with predictor with no ordinal constraints; $\mathcal{M}_{p+\beta}$ refers

to the model where all residuals, $\nu_{i}$, are constrained to be positive; and $\mathcal{M}_{(p+\beta) p}$ refers to the model were in addition the marginal study effects, $\theta_{i}$ are constrained to be positive with the predictor taken into account. Figure 5B highlights the distinction between the latter two models: The dashed line is $x_{i} \beta$, the standardized regression line, and the red lines represent $\nu_{i}$ for each study. To compare $\mathcal{M}_{u+\beta}$ and $\mathcal{M}_{p+\beta}$, we assess if all red lines go upward from the dashed line, that is, if all $\nu_{i}>0$. To compare $\mathcal{M}_{p+\beta}$ and $\mathcal{M}_{(p+\beta) p}$ we additionally assess if all blue points, the marginal study effects, are positive, that is, if all $\theta_{i}>0$.

The Bayes factor analysis shows that the preferred model remains the positive-effects 
A.

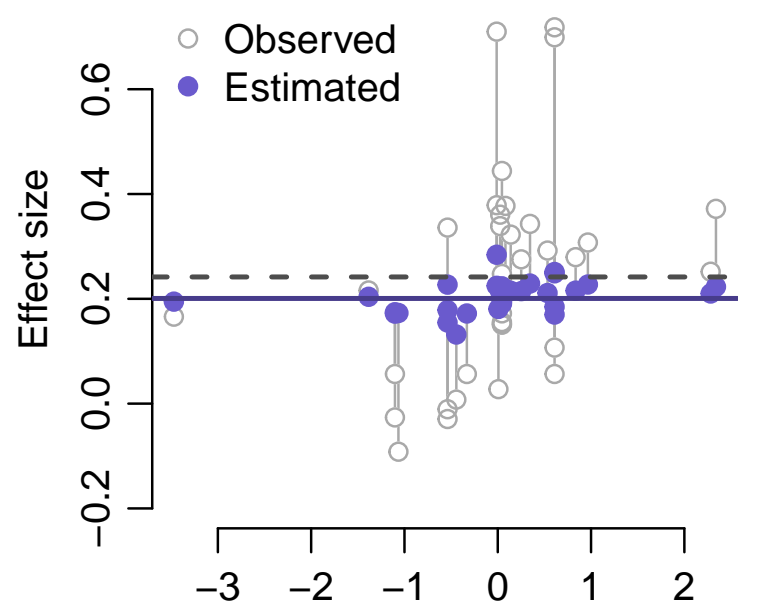

Standardized Average Age
B.

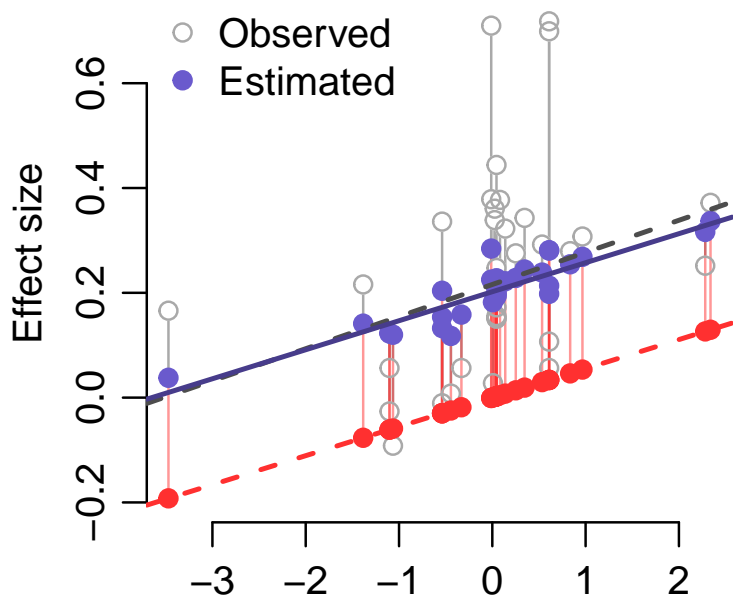

Standardized Average Age

Figure 5. Comparison of the meta-regression and the intercept-only analysis of age. The $\mathrm{x}$-axes show the standardized average age per study, the y-axes show Fisher's Z effect size estimates. Gray open points are observed effect sizes; blue points are estimated effect sizes from the Bayesian models. A. Results for the intercept-only model. The dashed line corresponds to the average observed effect size; the blue line corresponds to the estimated mean from the Bayesian model. B. Results for the meta-regression model. The dashed line corresponds to a simple least squares regression line; the blue line corresponds to the estimated regression line from the Bayesian meta-regression model. The red dashed line and points denote $x_{i} \beta$, the red vertical lines denote the non-zero-centered residuals $\nu_{i}$.

model without predictor. Model $\mathcal{M}_{u+\beta}$, performs poorest with a Bayes factor of 58.49-to-one in favor of the positive-effects model. Model $\mathcal{M}_{p+\beta}$, on the other hand, is the third-best model after the positive-effects and the common-effect models, with a Bayes factor of 3.67-toone in favor of the positive-effects model. We note that the best-performing model remains the positive-effects model. Hence, there is no evidence for the need to add the predictor.

\section{Critical Predictors}

As the replication crisis has roiled psychology, there have been a number of prominent failures to replicate previously established results (e.g. Open Science Collaboration, 2015; Ebersole et al., 2016). One popular set of beliefs is that the original results are in error, that is, the effects in question do not exist. But another popular set of beliefs is that perhaps both sets of results are correct - that is, the original results correctly showed an effect and 
Table 1

Bayes factors for the familiar-word-recognition meta-analysis.

\begin{tabular}{ll}
\hline Model & Bayes factor \\
\hline $\mathcal{M}_{u+\beta}$ & 0.27 \\
$\mathcal{M}_{p+\beta}$ & 4.26 \\
$\mathcal{M}_{(p+\beta) p}$ & 1.8 \\
$\mathcal{M}_{u}^{*}$ & 1 \\
$\mathcal{M}_{p}$ & 15.67 \\
$\mathcal{M}_{c}$ & 5.27 \\
$\mathcal{M}_{0}$ & 1.69 \\
\hline
\end{tabular}

Note. Bayes factors are in comparison to the unconstrained model.

the replications correctly showed a lack of one. The argument is that there must be some critical differences in the studies - critical hidden predictors that explain when the effect occurs and when it does not (Monin et al., 2014). The goal then is to find the missing critical predictors.

We are not fans of this approach, and were predisposed to think it is a waste of time. Nonetheless, the does-every-study approach with predictors may be easily adapted to assess the conjecture that a critical predictor explains effects and their failures on a study-by-study basis. In the following we develop the model for the conjecture. Then, we apply it to the familiar-word-recognition example for a predictor that the original authors speculated explained the effect. To our surprise, indeed, this predictor is critical and explains when the effect occurs and when it does not. Unfortunately, the unavoidable conclusion is that the effect may merely be an artifact of publication bias.

\section{The Critical Predictor Some-studies Model}

For this approach we ask whether a categorical variable predicts whether a study has a positive effect, or, alternatively, no effect at all. For example, we may want to assess the effect of preregistration on effect size under the hypothesis that preregistered studies have 
no effect and unregistered studies have an effect. Alternatively, we might think that there is an effect for one experimental procedure but not for the other. We refer to this setup as a some-studies model, and in it the categorical variable is manifest.

With the base model from Equation (3) the some-studies model can be placed on $\theta_{i}$ :

$$
\mathcal{M}_{s s}: \begin{cases}\theta_{i} \stackrel{i i d}{\sim} \operatorname{Normal}^{+}\left(\mu, \tau^{2}\right) & \text { if } x_{i}=1 \\ \theta_{i}=0 & \text { if } x_{i}=0\end{cases}
$$

where $x_{i}$ is an indicator variable for the dichotomous predictor at hand. Note that this model is quite constrained: Not only does it predict the direction of the effect for some studies, it also predicts zero-effects for other studies, and it a priori assigns each study to either of the two categories.

In the meta-analysis of familiar-word-recognition we consider a predictor called study use. Study use refers to whether the familiar-word-recognition effect was used as the target of study or as a baseline when exploring another effect. Through correspondence the original authors (Carbajal et al., 2021) hypothesized that, because data collection in developmental psychology is costly, the data were published irrespective of the outcome if the familiarword-recognition effect was the target of interest. Conversely, if the effect was considered a baseline it would perhaps be omitted from publication if the outcome was a null finding. As a result, the familiar-word-recognition effect may be inflated for studies where it was considered a baseline effect. We test Carbajal et al.'s conjecture here.

Figure 6 shows effect size estimates from the model for 32 of the 34 studies where it was clear whether the familiar-word-recognition effect was either target or baseline. In the majority of the studies with small effects the familiar-word-recognition effect was the main target of interest; in the majority of the studies with larger effects the familiar-wordrecognition effect was the baseline. We compared the some-studies model with the models 


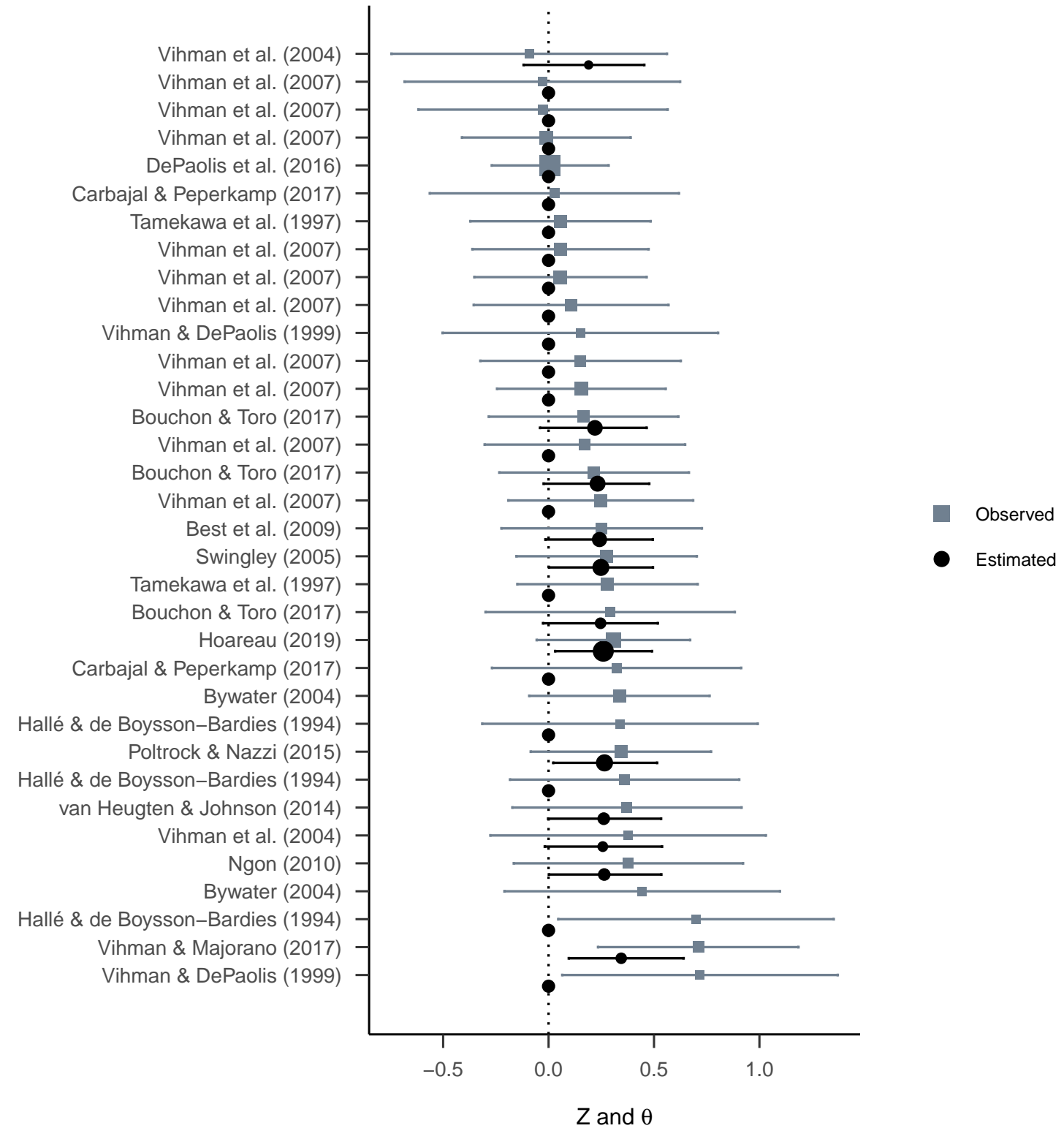

Figure 6. Estimates for the covariate model with study type as some-studies covariate. The black points show estimated study effects from the model where some studies have a zero effect as predicted by the moderator (familiar-word-recognition effect as baseline) and some studies have positive effects as predicted by the moderator (familiar-word-recognition effect as main target of interest). For two studies (Bywater, 2004) the moderator was not reported. 
Table 2

Bayes factors for the

target vs. baseline study

analysis.

\begin{tabular}{ll}
\hline Model & Bayes factor \\
\hline $\mathcal{M}_{u} *$ & 1 \\
$\mathcal{M}_{s s}$ & 141.31 \\
$\mathcal{M}_{p}$ & 13.37 \\
$\mathcal{M}_{c}$ & 5.64 \\
$\mathcal{M}_{0}$ & 2.31 \\
\hline
\end{tabular}

Note. Bayes factors are in comparison to the unconstrained model.

from the previous sections using Bayes factors (See Table 2). ${ }^{4}$ In accordance with Carbajal et al's conjecture, the some-studies model outperforms all the other models, and it is preferred to the positive-effects model by about 11 to 1 .

\section{Software}

The analyses presented here are conducted in $\mathrm{R}\left(\mathrm{R}\right.$ Core Team, 2016). ${ }^{5}$ As we hope this development is attractive to many researchers we took the following steps to make the adoption convenient. First, we provide an R-script containing all necessary functions to compute posterior estimates and Bayes factors at github. Second, we developed the Gibbs sampling functions that can be used on new data. ${ }^{6}$ The posterior distribution for models without predictors are estimated using the function mcmc_unconstrained(); the posterior

\footnotetext{
${ }^{4}$ The Bayes factors for the models shown both in Table 1 and Table 2 deviate slightly because less studies are included for Table 2 .

${ }^{5}$ The following packages were used: $\mathrm{R}$ (Version 4.0.3; R Core Team, 2016) and the R-packages abind (Version 1.4.5; Plate \& Heiberger, 2016), BayesFactor (Version 0.9.12.4.2; Morey \& Rouder, 2018), circular (Version 0.4.93; Agostinelli \& Lund, 2017), coda (Version 0.19.4; Plummer, Best, Cowles, \& Vines, 2006), fields (Version 10.3; Douglas Nychka, Reinhard Furrer, John Paige, \& Stephan Sain, 2017), ggplot2 (Version 3.3.3; Wickham, 2016), knitr (Version 1.33; Xie, 2015), magrittr (Version 2.0.1; Bache \& Wickham, 2014), MASS (Version 7.3.53; Venables \& Ripley, 2002), Matrix (Version 1.2.18; Bates \& Maechler, 2018), MCMCpack (Version 1.5.0; Martin, Quinn, \& Park, 2011), metafor (Version 3.0.1; Viechtbauer, 2010), msm (Version 1.6.8; Jackson, 2011), mvtnorm (Version 1.1.1; Genz \& Bretz, 2009; Wilhelm \& G, 2015), papaja (Version 0.1.0.9997; Aust \& Barth, 2017), psych (Version 2.1.3; Revelle, 2018), scam (Version 1.2.6; Pya, 2020), spatialfil (Version 0.15; Dinapoli \& Gatta, 2015), tmvtnorm (Version 1.4.10; Wilhelm \& G, 2015), and truncdist (Version 1.0.2; Novomestky \& Nadarajah, 2016).

${ }^{6}$ Some of the analyses provided here are also supported by the R-package metaBMA (Heck, Gronau, \& Wagenmakers, 2019).
} 
Table 3

Prior sensitivity analysis.

\begin{tabular}{llllll}
\hline Setting $c_{1}$ & Setting $c_{2}$ & $B F_{s s u}$ & $B F_{s s p}$ & $B F_{s s c}$ & $B F_{s s 0}$ \\
\hline 0.15 & 0.15 & 141.31 & 10.57 & 25.04 & 61.30 \\
0.30 & 0.30 & $4,527.52$ & 908.46 & 102.30 & 15.94 \\
0.08 & 0.30 & 419.63 & 121.43 & 117.09 & 18.24 \\
0.30 & 0.08 & $2,182.60$ & 3.58 & 137.34 & 345.19 \\
0.08 & 0.08 & 35.98 & 1.89 & 15.58 & 39.17 \\
\hline
\end{tabular}

distribution for models with predictors are estimated using the function mcmc_predictor(); and the posterior distribution for models without critical predictors are estimated using the function mcmc_somesstudies(). Functions get.bfs.ss() and get.bfs.cont() provide Bayes factor estimation for all proposed models. The analysis of the familiar-word-recognition effect (Carbajal et al., 2021) is provided at github as well.

\section{Prior Sensitivity}

Bayes factor model comparison requires the choice of settings on prior distributions. Some prior settings have minimal influence on the results of the analysis while others have substantial influence. In the current analysis the settings of $c_{1}$ and $c_{2}$ have the largest influence. One way of assessing the effect of the prior choices on inference is to conduct a sensitivity analyses. In a sensitivity analysis, prior settings are adjusted within reasonable ranges and the resulting variability of Bayes factors is assessed (e.g., Haaf \& Rouder, 2017; Heycke, Gehrmann, Haaf, \& Stahl, 2018). Here, we conduct a sensitivity analysis for the familiar-word-recognition effect. We chose to double and halve the scale on $\mu, c_{1}$, and the scale on $\tau, c_{2}$ from our original setting (between 0.08 and 0.30 ).

Table 3 shows the Bayes factors for the original analysis (top row) and four additional analyses with varied settings. Bayes factors are between the preferred some-studies model and the unconstrained, positive-effects, common-effect, and null models, respectively. If all values in a row remain above 1 it implies that the some-studies model is superior for the particular prior setting. However, if a values is below 1 it implies that an alternative 
model is superior, and therefore the inference is not robust to the chosen prior settings. For the data at hand, while Bayes factors vary considerably, the some-studies model is preferred for all settings in the sensitivity analysis. The pattern of Bayes factors is fairly straight-forward: For smaller scale settings the Bayes factor between the positive-effects model and the some-studies model is relatively close to 1 ; for larger scales the null model is actually preferred over the positive-effects model, and this phenomenon is well-understood as Lindley's paradox (Lindley, 1957). Yet, within these reasonable ranges of prior settings, the some-studies model is robustly preferred over all of its alternatives.

\section{Discussion}

Meta-analysis is a powerful tool to summarize evidence across several studies. In the absence of the raw data it is the most straight-forward method to combine quantitative results across multiple studies. Here, we address the fundamental issue in meta-analysis of what forms an appropriate summary across studies. Rather than focusing on an overall effect size, we ask whether every study in the data set plausibly shows an effect in the expected direction. The current approach is motivated by the presence of coarse theory in psychology and the desire for robustness and replicability across many design elements. When theory is coarse, it provides at most an ordinal prediction on effects (Haaf et al., 2019). If all studies plausibly show a true effect in the same direction, then the phenomenon may robustly be found across varying operationalizations and study settings. To answer the question whether every study shows a true effect in the same direction we develop a set of models: A general model much like the conventional meta-analytic random-effects model; a positive-effects model where all studies have a true effect in the same direction; a common-effect model where all true effects are the same; and a null model where all true effects are identically zero. To assess the relative strength of evidence between these models, we propose a Bayes factor model comparison approach adapted from Haaf and Rouder (2017) and Rouder et al. (2019). The crucial innovation here is that the new approach takes summary statistics from each study instead of raw data. 
A second scientific question for meta-analysis concerns the role of predictor variables. We develop a new set of models that describe how covariates affect the direction of effects. We

apply the does-every-study meta-analysis to the familiar-word-recognition effect (Carbajal et al., 2021) in babies. In developmental research, small sample sizes are common, and meta-analysis therefore remains a central methodological approach. The results from our analysis provide evidence for the claim that studies either show a true positive effect, or truly no effect whatsoever. We did not find a qualitative interaction where some studies truly show an opposite effect. Importantly, there seems to be a critical predictor that determines whether a study will show an effect or not. Studies designed to test the effect result in null effects. Those that use the effect incidentally show positive effects. We suspect publication bias when the effect is not the target of the study.

\section{Limitations and Future Directions}

With every model development come model assumptions that need to be taken into account. The advantage of the current approach is that it only requires surface summary statistics. However, modeling these surface statistics introduces the assumption that the observed within-study variance is the true variance. This assumption is actually common in many linear modeling setups such as the computation of BIC. How good of an assumption it is is simply a function of the size of the sample. With large sample sizes obviously the observed variance is very close to the true one. With smaller studies such as in developmental psychology, however, the true variance might be quite different.

Another limitation of the current approach is that, except in some cases, it does take publication bias into account. Although we use the some-studies approach to identify a critical predictor we speculate that publication bias plays a role without actually modeling it. Yet, publication bias and questionable research practices are intimately tied to the question of the utility of meta-analysis (Carter, Schönbrodt, Hilgard, \& Gervais, 2017; Corker, 2018). If the studies in the meta-analytic set are heavily biased, using them to learn about the population is difficult. 
One of the main innovations in meta-analysis is the proposal of models and methods to correct for publication bias (Duval \& Tweedie, 2000; Hedges, 1984; Iyengar \& Greenhouse, 1988; Simonsohn, Nelson, \& Simmons, 2014; Stanley \& Doucouliagos, 2014). One possible extension of the current modeling approach is to model selection effects simultaneously with true effects (Iyengar \& Greenhouse, 1988; Maier, Bartoš, \& Wagenmakers, 2020). In principle, this extension should not be too difficult. Yet, assessing the success of these corrections can be problematic as neither the process of study censorship nor the amount of publication bias are known in any real meta-analytic set (Guan \& Vandekerckhove, 2016). One additional difficulty here is the role of skewness of the distribution of observed effects. In the current approach, skewness comes from an underlying skewed distribution of true effects; in most publication bias correction models, in contrast, skewness reflects the censoring

process. While it may be possible to disentangle publication bias and true positive effects by assessing the relationship of sample size and effect size, a robust, general solution remains an open issue.

A third limitation of the current approach concerns the type of predictors considered in the meta-regression analysis. Here, we only developed a framework for study-level predictors. However, it is increasingly common to assess the effect of within-study predictors such as age per study condition, or different dependent variables assessed within the same sample. If such within-study predictors are included, a three-level meta-analysis has to be conducted to account for the within-study dependency (Cheung, 2014; Van den Noortgate, López-López, Marín-Martínez, \& Sánchez-Meca, 2013). One future improvement may be the extension of every-study-does meta-analysis to three-level meta-analysis.

\section{Conclusion}

In summary, the current approach allows to investigate questions of ordinal constraint in a meta-analytic setting. We think that answering the does-every-study question is timely and topical. If, indeed, every study shows a true effect in the same, expected direction, maintaining an underlying theory for the target phenomenon seems reasonable. If, however, 
the ordinal constraint is violated and some studies show true opposite effects, researchers need to investigate the underlying mechanism that lead to this qualitative interaction. These mechanisms may be found in design or population, and the proposed predictor analysis can help understanding the underlying differences. 


\section{References}

Agostinelli, C., \& Lund, U. (2017). R package circular: Circular statistics (version 0.4-93). CA: Department of Environmental Sciences, Informatics; Statistics, Ca' Foscari University, Venice, Italy. UL: Department of Statistics, California Polytechnic State University, San Luis Obispo, California, USA. Retrieved from https://r-forge.rproject.org/projects/circular/

Aust, F., \& Barth, M. (2017). papaja: Create APA manuscripts with R Markdown. Retrieved from https://github.com/crsh/papaja

Bache, S. M., \& Wickham, H. (2014). Magrittr: A forward-pipe operator for $r$. Retrieved from https://CRAN.R-project.org/package=magrittr

Bates, D., \& Maechler, M. (2018). Matrix: Sparse and dense matrix classes and methods. Retrieved from https://CRAN.R-project.org/package=Matrix

Borenstein, M., Cooper, H., Hedges, L., \& Valentine, J. (2009). Effect sizes for continuous data. The Handbook of Research Synthesis and Meta-Analysis, 2, 221-235.

Borenstein, M., Hedges, L. V., Higgins, J. P., \& Rothstein, H. R. (2011). Introduction to meta-analysis. John Wiley \& Sons.

Borenstein, M., Hedges, L. V., Higgins, J., \& Rothstein, H. R. (2010). A basic introduction to fixed-effect and random-effects models for meta-analysis. Research Synthesis Methods, 1(2), 97-111.

Carbajal, M. J., Peperkamp, S., \& Tsuji, S. (2021). A meta-analysis of infants' word-form recognition. Infancy. Retrieved from https://doi.org/10.1111/infa.12391

Carter, E. C., Schönbrodt, F. D., Hilgard, J., \& Gervais, W. M. (2017). Correcting for bias in psychology: A comparison of meta-analytic methods. Retrieved from https://osf.io/preprints/psyarxiv/9h3nu/

Cheung, M. W.-L. (2014). Modeling dependent effect sizes with three-level meta-analyses: 
A structural equation modeling approach. Psychological Methods, 19(2), 211-229.

Corker, K. S. (2018). Strengths and weaknesses of meta-analyses. Retrieved from https: //psyarxiv.com/6gcnm/

Corker, K. S., Donnellan, M. B., Kim, S. Y., Schwartz, S. J., \& Zamboanga, B. L. (2017). College student samples are not always equivalent: The magnitude of personality differences across colleges and universities. Journal of Personality, 85(2), 123-135.

Dinapoli, N., \& Gatta, R. (2015). Spatialfil: Application of $2 D$ convolution kernel filters to matrices or $3 D$ arrays. Retrieved from https://CRAN.R-project.org/package= spatialfil

Douglas Nychka, Reinhard Furrer, John Paige, \& Stephan Sain. (2017). Fields: Tools for spatial data. Boulder, CO, USA: University Corporation for Atmospheric Research. doi:10.5065/D6W957CT

Duval, S., \& Tweedie, R. (2000). Trim and fill: A simple funnel-plot-based method of testing and adjusting for publication bias in meta-analysis. Biometrics, 56, 455-463. Retrieved from 10.1111/j.0006-341X.2000.00455.x

Ebersole, C. R., Atherton, O. E., Belanger, A. L., Skulborstad, H. M., Allen, J. M., Banks, J. B., ... Nosek, B. A. (2016). Many labs 3: Evaluating participant pool quality across the academic semester via replication. Journal of Experimental Social Psychology, 67, 68-82. Retrieved from http://ezid.cdlib.org/id/doi:10.17605/OSF.IO/QGJM5

Erp, S. van, Verhagen, J., Grasman, R. P., \& Wagenmakers, E.-J. (2017). Estimates of between-study heterogeneity for 705 meta-analyses reported in psychological bulletin from 1990-2013. Journal of Open Psychology Data, 5(1).

Ferguson, T. S. (1996). A course in large sample theory. Chapman \& Hall Ltd.

Gail, M., \& Simon, R. (1985). Testing for qualitative interactions between treatment effects and patient subsets. Biometrics, 41(2), 361-372. 
Gayen, A. K. (1951). The frequency distribution of the product-moment correlation coefficient in random samples of any size drawn from non-normal universes. Biometrika, 38(1/2), $219-247$.

Genz, A., \& Bretz, F. (2009). Computation of multivariate normal and $t$ probabilities. Heidelberg: Springer-Verlag.

Gronau, Q. F., Ly, A., \& Wagenmakers, E.-J. (2019). Informed bayesian t-tests. The American Statistician.

Guan, M., \& Vandekerckhove, J. (2016). A Bayesian approach to mitigation of publication bias. Psychonomic Bulletin and Review, 23(1), 74-86. Retrieved from http://www. cidlab.com/prints/guan2015bayesian.pdf

Haaf, J. M., Klaassen, F., \& Rouder, J. N. (2019). Capturing ordinal theoretical constraint in psychological science. Retrieved from https://psyarxiv.com/a4xu9/

Haaf, J. M., \& Rouder, J. N. (2017). Developing constraint in Bayesian mixed models. Psychological Methods, 22(4), 779-798.

Haaf, J. M., \& Rouder, J. N. (2019). Some do and some don't? Accounting for variability of individual difference structures. Psychonomic Bulletin and Review, 26, 772-789. Retrieved from https://doi.org/10.3758/s13423-018-1522-x

Hallé, P. A., \& Boysson-Bardies, B. de. (1994). Emergence of an early receptive lexicon: Infants' recognition of words. Infant Behavior and Development, 17(2), 119-129.

Heck, D., Gronau, Q., \& Wagenmakers, E. (2019). MetaBMA: Bayesian model averaging for random and fixed effects meta-analysis. Retrieved from https://github.com/danheck/ metaBMA

Hedges, L. V. (1984). Estimation of effect size under nonrandom sampling: The effects of censoring studies yielding statistically insignificant mean differences. Journal of Educational Statistics, 9(1), 61-85. 
Hedges, L. V., \& Vevea, J. L. (1998). Fixed-and random-effects models in meta-analysis. Psychological Methods, 3(4), 486.

Heycke, T., Gehrmann, S., Haaf, J. M., \& Stahl, C. (2018). Of two minds or one? A registered replication of rydell et al.(2006). Cognition and Emotion, 0(0), 1-20.

Higgins, J. P., Thompson, S. G., \& Spiegelhalter, D. J. (2009). A re-evaluation of randomeffects meta-analysis. Journal of the Royal Statistical Society: Series A (Statistics in Society), 172(1), 137-159.

Hoijtink, H. (2012). Informative Hypotheses. Theory and Practice for Behavioral and Social Scientists. Boca Raton: Chapman \& Hall/CRC.

Iyengar, S., \& Greenhouse, J. B. (1988). Selection models and the file drawer problem. Statistical Science, 109-117.

Jackson, C. H. (2011). Multi-state models for panel data: The msm package for R. Journal of Statistical Software, 38(8), 1-29. Retrieved from http://www.jstatsoft.org/v38/i08/

Jeffreys, H. (1961). Theory of probability (3rd edition). New York: Oxford University Press.

Kass, R. E., \& Raftery, A. E. (1995). Bayes factors. Journal of the American Statistical Association, 90, 773-795. Retrieved from http://amstat.tandfonline.com/doi/abs/10. 1080/01621459.1995.10476572

Klugkist, I., Laudy, O., \& Hoijtink, H. (2005). Inequality constrained analysis of variance: A Bayesian approach. Psychological Methods, 10(4), 477.

Lindley, D. V. (1957). A statistical paradox. Biometrika, 44, 187-192.

Maier, M., Bartoš, F., \& Wagenmakers, E.-J. (2020). Robust bayesian meta-analysis: Addressing publication bias with model-averaging.

Martin, A. D., Quinn, K. M., \& Park, J. H. (2011). MCMCpack: Markov chain monte carlo in R. Journal of Statistical Software, 42(9), 22. Retrieved from http://www.jstatsoft. $\operatorname{org} / \mathrm{v} 42 / \mathrm{i} 09 /$ 
Monin, B., Oppenheimer, D. M., Ferguson, M. J., Carter, T. J., Hassin, R. R., Crisp, R. J., ... others. (2014). Commentaries and rejoinder on klein et al.(2014). Social Psychology, 299-311.

Morey, R. D., Romeijn, J.-W., \& Rouder, J. N. (2016). The philosophy of Bayes factors and the quantification of statistical evidence. Journal of Mathematical Psychology, 72, 6-18. Retrieved from http://www.sciencedirect.com/science/article/pii/ S0022249615000723

Morey, R. D., \& Rouder, J. N. (2018). BayesFactor: Computation of bayes factors for common designs. Retrieved from https://CRAN.R-project.org/package=BayesFactor

Novomestky, F., \& Nadarajah, S. (2016). Truncdist: Truncated random variables. Retrieved from https://CRAN.R-project.org/package=truncdist

Open Science Collaboration. (2015). Estimating the reproducibility of psychological science. Science, 349(6521), 943. Retrieved from dx.doi.org/10.1126/science.aac4716

Pan, G., \& Wolfe, D. A. (1997). Test for qualitative interaction of clinical significance. Statistics in Medicine, 16(14), 1645-1652.

Plate, T., \& Heiberger, R. (2016). Abind: Combine multidimensional arrays. Retrieved from https://CRAN.R-project.org/package=abind

Plummer, M., Best, N., Cowles, K., \& Vines, K. (2006). CODA: Convergence diagnosis and output analysis for mcmc. $R$ News, 6(1), 7-11. Retrieved from https://journal.rproject.org/archive/

Pya, N. (2020). Scam: Shape constrained additive models. Retrieved from https://CRAN.Rproject.org $/$ package $=$ scam

R Core Team. (2016). R: A language and environment for statistical computing. Vienna, Austria: R Foundation for Statistical Computing. Retrieved from https://www.Rproject.org/ 
Revelle, W. (2018). Psych: Procedures for psychological, psychometric, and personality research. Evanston, Illinois: Northwestern University. Retrieved from https://CRAN. R-project.org $/$ package $=$ psych

Rouder, J. N., Haaf, J. M., \& Aust, F. (2018). From theories to models to predictions: A Bayesian model comparison approach. Communication Monographs, 85, 41-56. Retrieved from https://doi.org/10.1080/03637751.2017.1394581

Rouder, J. N., Haaf, J. M., Davis-Stober, C. P., \& Hilgard, J. (2019). Beyond overall effects: A Bayesian approach to finding constraints in meta-analysis. Psychological Methods.

Rouder, J. N., \& Morey, R. D. (2019). Teaching bayes' theorem: Strength of evidence as predictive accuracy. The American Statistician, 73, 186-190. Retrieved from https://doi.org/10.1080/00031305.2017.1341334

Rouder, J. N., Morey, R. D., Speckman, P. L., \& Province, J. M. (2012). Default Bayes factors for ANOVA designs. Journal of Mathematical Psychology, 56, 356-374. Retrieved from http://dx.doi.org/10.1016/j.jmp.2012.08.001

Rouder, J. N., Morey, R. D., \& Wagenmakers, E.-J. (2016). The interplay between subjectivity, statistical practice, and psychological science. Collabra, 2, 6. Retrieved from http://doi.org/10.1525/collabra.28

Rouder, J. N., Speckman, P. L., Sun, D., Morey, R. D., \& Iverson, G. (2009). Bayesian t-tests for accepting and rejecting the null hypothesis. Psychonomic Bulletin and Review, 16, 225-237. Retrieved from http://dx.doi.org/10.3758/PBR.16.2.225

Schlattmann, P. (2009). Medical applications of finite mixture models. Springer.

Simonsohn, U., Nelson, L. D., \& Simmons, J. P. (2014). P-curve: A key to the filedrawer. Journal of Experimental Psychology: General, 143, 534-547. Retrieved from $10.1037 / \mathrm{a} 0033242$

Stanley, T. D., \& Doucouliagos, H. (2014). Meta-regression approximations to reduce 
publication selection bias. Research Synthesis Methods, 5(1), 60-78. Retrieved from DOI: $10.1002 / j$ jrsm.1095

Thiele, J. E., Haaf, J. M., \& Rouder, J. N. (2017). Bayesian analysis for systems factorial technology. Journal of Mathematical Psychology, 81, 40-54.

Van den Noortgate, W., López-López, J. A., Marín-Martínez, F., \& Sánchez-Meca, J. (2013). Three-level meta-analysis of dependent effect sizes. Behavior Research Methods, $45(2), 576-594$.

Vanpaemel, W. (2010). Prior sensitivity in theory testing: An apologia for the Bayes factor. Journal of Mathematical Psychology, 54, 491-498.

Vanpaemel, W., \& Lee, M. D. (2012). Using priors to formalize theory: Optimal attention and the generalized context model. Psychonomic Bulletin \& Review, 19, 1047-1056.

Venables, W. N., \& Ripley, B. D. (2002). Modern applied statistics with s (Fourth.). New York: Springer. Retrieved from http://www.stats.ox.ac.uk/pub/MASS4

Viechtbauer, W. (2010). Conducting meta-analyses in R with the metafor package. Journal of Statistical Software, 36(3), 1-48. Retrieved from http://www.jstatsoft.org/v36/i03/

Wagenmakers, E.-J., Beek, T., Dijkhoff, L., Gronau, Q. F., Acosta, A., R. B. Adams, J., ... Zwaan, R. A. (2016). Registered replication report: Strack, martin, \& stepper (1988). Perspectives on Psychological Science, 11(6), 917-928. Retrieved from https://doi.org/10.1177/1745691616674458

Wickham, H. (2016). Ggplot2: Elegant graphics for data analysis. Springer-Verlag New York. Retrieved from http://ggplot2.org

Wilhelm, S., \& G, M. B. (2015). tmvtnorm: Truncated multivariate normal and student $t$ distribution. Retrieved from http://CRAN.R-project.org/package=tmvtnorm

Xie, Y. (2015). Dynamic documents with $R$ and knitr (2nd ed.). Boca Raton, Florida: Chapman; Hall/CRC. Retrieved from https://yihui.name/knitr/ 


\section{Online Supplement}

The asymptotic distribution of $Z$ is well established for the correlation between two independent and identically distributed normal random variables (Ferguson, 1996). In the case of correlation, Fisher's $Z$ serves as normalized correlation measure. Yet, how does Fisher's $Z$ translate to experimental data? Let's assume an experimental setup with $j$ conditions, $j=1,2$. The $i$ th participant is assigned to one of the two conditions, $i=1, \ldots, 2 n$, where $n$ is the number of participants in each condition and $2 n$ is the total number of participants. Then $Y_{i}$ denotes the $i$ th person's observation with

$$
Y_{i} \sim \begin{cases}\operatorname{Normal}\left(\mu_{1}, \sigma^{2}\right) & \text { if } i=1, \ldots, n, \\ \operatorname{Normal}\left(\mu_{2}, \sigma^{2}\right) & \text { if } i=n+1, \ldots, 2 n,\end{cases}
$$

where $\mu_{j}$ denotes the true mean of the $j$ th condition and $\sigma^{2}$ denotes the within-group variance. To calculate Fisher's $Z$ as effect size measure we could first calculate Cohen's $d$ and then use common transformations via the correlation coefficient $r$. To understand what this correlation coefficient represents we may define a new variable, $X_{i}$, that denotes $i$ th participant's condition. $X_{i}$ is an effect variable encoding $j=1$ as $X_{i}=-1$ and $j=2$ as $X_{i}=1$. The correlation coefficient $r$ is the point-biserial correlation between $\mathbf{X}$ and $\mathbf{Y}$, and Fisher's $Z$ may be calculated using Equation (1). Yet, we need to verify that the asymptotic distribution of Fisher's $Z$ for the biserial correlation is the same as the asymptotic distribution of Fisher's $Z$ for the bivariate normal. Here, we provide a proof that the asymptotic variance is stable and does not depend on the size of the effect.

Let $\bar{Y}$ be the grand mean of $\mathbf{Y}, \bar{Y}=1 / 2\left(\bar{Y}_{1}+\bar{Y}_{2}\right)$. Here, $\bar{Y}_{1}$ denotes the mean of $Y_{i}$ for $i=1, \ldots, n$, and $\bar{Y}_{2}$ denotes the mean of $Y_{i}$ for $i=n+1, \ldots, 2 n$. Let $S_{Y Y}$ denote the variance of $\mathbf{Y}, S_{Y Y}=\sum_{1}^{n}\left(Y_{i}-\bar{Y}_{1}\right)^{2}+\sum_{n+1}^{2 n}\left(Y_{i}-\bar{Y}_{2}\right)^{2}+n / 2\left(\bar{Y}_{1}-\bar{Y}_{2}\right)^{2}=S S_{1}+S S_{2}+n / 2\left(\bar{Y}_{1}-\bar{Y}_{2}\right)^{2}$, where $S S_{1}$ is the sum of squares for $i=1, \ldots, n$, and $S S_{2}$ is the sum of squares for 
$i=n+1, \ldots, 2 n$. Let $S_{X X}=2 n$ denote the variance of $\mathbf{X}$ and let $S_{X Y}=n\left(\bar{Y}_{1}-\bar{Y}_{2}\right)$ denote the covariance of $\mathbf{X}$ and $\mathbf{Y}$. We can now insert these quantities into the formula for the correlation coefficient $r$ :

$$
\begin{aligned}
r & =\frac{S_{X Y}}{\sqrt{S_{X X} S_{Y Y}}} \\
& =\frac{\sqrt{1 / 2}\left(\bar{Y}_{1}-\bar{Y}_{2}\right)}{\sqrt{\left.\frac{2\left(S S_{1}+S S_{2}\right)}{2 n}+1 / 2\left(\bar{Y}_{1}-\bar{Y}_{2}\right)^{2}\right)}} .
\end{aligned}
$$

We may define a new random variable $m_{n}=\bar{Y}_{1}-\bar{Y}_{2}$. Then, based on the Central Limit Theorem:

$$
\sqrt{n}\left(m_{n}-\Delta\right) \stackrel{\mathscr{D}}{\rightarrow} \operatorname{Normal}\left(0,2 \sigma^{2}\right)
$$

where $\Delta$ is the true effect. The pooled variance of $\mathbf{Y}$ is $S_{n}=\frac{S S_{1}+S S_{2}}{2 n} .^{7}$ The Central Limit Theorem gives $\sqrt{n}\left(S_{n}-2 \sigma^{2}\right) \stackrel{\mathscr{D}}{\rightarrow} \operatorname{Normal}\left(0,4 \sigma^{2}\right)$. Using the two asymptotic distributions, $r \rightarrow \frac{\sqrt{1 / 2}(\Delta)}{\sqrt{4 \sigma^{2}+\Delta^{2} / 2}}$. Let $\Sigma$ be the variance-covariance matrix of $\left(\begin{array}{c}m_{n} \\ S_{n}\end{array}\right)$ :

$$
\Sigma=\left[\begin{array}{cc}
2 \sigma^{2} & 0 \\
0 & 4 \sigma^{2}
\end{array}\right]
$$

And let $h(a, b)=\frac{\sqrt{1 / 2}(a)}{\sqrt{2 b+a^{2} / 2}}$. The partial derivatives of $h(a, b)$ are:

$$
\begin{aligned}
\frac{\partial h}{\partial a} & =\frac{\sqrt{2} b}{\left(2 b+a^{2} / 2\right)^{3 / 2}}, \\
\frac{\partial h}{\partial b} & =-\frac{a}{\sqrt{2}\left(2 b+a^{2} / 2\right)^{3 / 2}} .
\end{aligned}
$$

\footnotetext{
${ }^{7}$ Note that $S_{n}$ is not the variance of $\mathbf{Y}$, but the pooled variance of $Y_{i}$ if $i=1, \ldots, n$ and $Y_{i}$ if $i=n+1, \ldots, 2 n$
} 
Using the Delta-method (Ferguson, 1996), we may express the asymptotic distribution of $h\left(m_{n}, S_{n}\right)$ as

$$
\sqrt{n}\left(h\left(m_{n}, S_{n}\right)-h\left(\Delta, 2 \sigma^{2}\right) \stackrel{\mathscr{D}}{\rightarrow} \operatorname{Normal}\left(0, \Sigma^{*}\right),\right.
$$

where $h\left(m_{n}, S_{n}\right)$ is the correlation coefficient, the mean, $h\left(\Delta, 2 \sigma^{2}\right)$, is the true correlation coefficient, $\rho$, and the asymptotic variance is

$$
\begin{aligned}
\Sigma^{*} & =\left(\frac{2 \sqrt{2} \sigma^{2}}{\left(4 \sigma^{2}+\Delta^{2} / 2\right)^{3 / 2}}\right)^{2} 2 \sigma^{2}+\left(\frac{-\Delta}{\sqrt{2}\left(4 \sigma^{2}+\Delta^{2} / 2\right)^{3 / 2}}\right)^{2} 4 \sigma^{2} \\
& =\frac{16}{\left(8+\frac{\Delta^{2}}{\sigma^{2}}\right)^{2}}
\end{aligned}
$$

We may apply Fisher's $Z$ transformation to stabilize the variance. Let $g(r)=$ $1 / 2 \log \left(\frac{1+r}{1-r}\right)$. Then $g^{\prime}(r)=\frac{1}{1-r^{2}}$. Substituting $h\left(\Delta, 2 \sigma^{2}\right)$ for $\rho, g^{\prime}(\rho)=\frac{8+\frac{\Delta^{2}}{\sigma^{2}}}{8}$. We may again use the Delta-method to express the asymptotic distribution of $Z$ :

$$
\sqrt{n}\left(g(r)-g(\rho) \stackrel{\mathscr{D}}{\rightarrow} \operatorname{Normal}\left(0,\left(g^{\prime}(\rho)\right)^{2} \Sigma^{*}\right)\right.
$$

where $g(r)=Z$ and $g(\rho)=\theta$. The asymptotic variance is

$$
\left(g^{\prime}(\rho)\right)^{2} \Sigma^{*}=\frac{\left(8+\frac{\Delta^{2}}{\sigma^{2}}\right)^{2}}{8^{2}} \frac{16}{\left(\underline{8}+\frac{\Delta^{2}}{\sigma^{2}}\right)^{2}}=1 / 4
$$

Crucially, this value, $1 / 4$, does not depend on $\rho$. 